\title{
Application of response surface methodology to tailor the surface chemistry of electrospun chitosan-poly(ethylene oxide) fibers
}

\author{
Peter Bösiger ${ }^{a, b}$, Isabelle M. T. Richard ${ }^{a, c}$, Luce LeGat ${ }^{a, d}$, Benjamin Michen ${ }^{e, f}$, Mark Schubert ${ }^{e}$, René \\ M. Rossia, Giuseppino Fortunato ${ }^{\mathrm{a},}$
}
a Empa, Laboratory for Biomimetic Membranes and Textiles, Lerchenfeldstrasse 5, CH-9014 St. Gallen, Switzerland.
b University of Freiburg, Faculty of Environment \& Natural Resources, Chair of Forest Botany, Ber- toldstrasse 17, DE-79085 Freiburg, Germany

c École européenne de Chimie, Polymères et Matériaux, Université de Strasbourg, 25 Rue Becquerel, FR-67087 Strasbourg, France

${ }^{d}$ CBMN (UMR 5248, CNRS), 3BIO's team, University of Bordeaux, Allée Geoffroy Saint-Hilaire, 33600 Pessac, France

e Empa, Laboratory for Applied Wood Materials, Überlandstrasse 129, CH-8600 Dübendorf, Switzerland.

${ }^{f}$ Wood Materials Science, Institute for Building Materials, ETH Zürich, Stefano-Franscini-Platz 3, 8093 Zürich, Switzerland

* corresponding author. E-mail address: giuseppino.fortunato@empa.ch

\section{Highlights}

- Development of electrospun chitosan / PEO blend fibers with tailored surface properties.

- Designing of a response surface model (RSM) exhibiting excellent prediction accuracy.

- Chitosan fibers exhibiting tailored nitrogen atomic surface content of up to $6.4 \%$.

- Nitrogen surface content is significantly determined by chitosan $\mathrm{Mw}$ and polymers mixing ratio.

- Nitrogen surface content strongly influences the membranes zeta potential.

This document is the accepted manuscript version of the following article:

Bösiger, P., Richard, I. M. T., LeGat, L., Michen, B., Schubert, M., Rossi, R. M., \& Fortunato, G. (2018). Application of response surface methodology to tailor the surface chemistry of electrospun chitosan-poly (ethylene oxide) fibers. Carbohydrate Polymers. http://doi .org/10.1016/j.carbpo1.2018.01.038 


\section{Abstract}

Chitosan is a promising biocompatible polymer for regenerative engineering applications, but its processing remains challenging due to limited solubility and rigid crystalline structure. This work represents the development of electrospun chitosan / poly(ethylene oxide) blend nanofibrous membranes by means of a numerical analysis in order to identify and tailor the main influencing parameters with respect to accessible surface nitrogen functionalities which are of importance for the biological activity as well as for further functionalization. Depending on the solution composition, both gradient fibers and homogenous blended fiber structures could be obtained with surface nitrogen concentrations varying between 0 and $6.4 \%$. Response surface methodology (RSM) revealed chitosan / poly(ethylene oxide) ratio and chitosan molecular weight as the main influencing factors with respect to accessible nitrogen surface atoms and respective concentrations. The model showed good adequacy hence providing a tool to tailor the surface properties of chitosan / poly(ethylene oxide) blends by addressing the amount of accessible chitosan.

Keywords: Chitosan, Electrospinning, Nanofibers, Gradient fibers, Response surface methodology 


\section{Introduction:}

Electrospun nonwovens provide unique features such as high surface to volume ratio, a structural resemblance to extracellular matrix (ECM) (Nisbet et al. 2009) and tailored porosity allowing for excellent mass transfer, making them promising candidates as scaffolds in regenerative medicine (Guex et al. 2012; Tchemtchoua et al. 2011) as well as for therapeutic drug delivery platforms (Yazgan et al. 2015; Ghitescu et al. 2015). The process of electrospinning is not only straightforward, cost-efficient and versatile in producing non-wovens made of nano- to micron-sized fibers, but it also allows for the systematic design of nonwovens with defined characteristics i.e. specifically for the controlled release of bioactive compounds, an improved cellular response and bio-compatibility (Nisbet et al. 2009) or optimized degradation profiles (Yang et al. 2007; Kishan et al. 2015).

Chitosan is a widely abundant low cost poly-cationic biopolymer, which is often used for tissue engineering applications owing to its manifold proven bioactivity combined with non-toxicity, proven cytocompatibility towards various cell types (Tchemtchoua et al. 2011; Ajalloueian et al. 2014) and in vivo biodegradability. As chitosan forms strong intra- and inter-molecular hydrogen bonds, it is often blended with other polymers of natural or synthetic origin for better processability into homogeneous electrospun fibers (Pakravan et al. 2011). Hybrid scaffolds previously reported include mixtures of chitosan with polylactic acid (PLA), polyvinyl alcohol (PVA), polycaprolactone ( $P C L)$ polyethylene oxide (PEO), gelatin, zein, silk fibroin or alginate. These secondary polymers can positively influence fiber properties such as mechanical strength, swelling behavior and biocompatibility of the hybrid structure for applications as antimicrobial dressings, drug delivery systems and in tissue engineering scaffolds (Pakravan et al. 2012a).

PEO improves the stability of the spinning process by increasing the chain entanglement of chitosan while decreasing the electrical conductivity of the spinning solution (Pakravan et al. 2011; Spasova et al. 2004; Li \& Hsieh 2006; Duan et al. 2004). The incorporation of high molecular weight PEO has been shown to facilitate fiber formation in particular, and at remarkably low concentration ranges (Zhang et al. 2008). As a result of its strong biocompatibility and the approval for internal use in food, cosmetics, personal care products and for pharmaceuticals (Bhattarai et al. 2005), the blended membranes are not subject to any constraints in biomedical applications. 
Through spinning homogeneous $\mathrm{PEO} /$ chitosan solutions in a non-coaxial setup, various fiber morphologies have been obtained. These include core-shell structures (Zhang et al. 2009), fully homogenous fibers with a uniform internal structure (Zhang et al. 2008), as well as bimodal distributions of ultrafine chitosan and micron-sized PEO fibers (Duan et al. 2004). As a consequence of these different morphologies, the nitrogen surface composition deriving from chitosan does not necessarily reflect the bulk composition and is highly dependent on both solution and spinning parameters. This is of importance for the chitosan non-wovens, as the free amine groups are important for crosslinking procedures and for further functionalization ( $\mathrm{Li}$ et al. 2012; Tegl et al. 2016; Croce et al. 2016). Furthermore, in an acidic environment these amine groups become protonated (pKa in the range of 6.17-6.51 (Wang et al. 2006)) turning chitosan into a polycationic polymer. Surface charges influence the degree of biomolecular affinity on a material surface via electrostatic interaction, enabling the production of tailor made polymer surfaces. Surface charged materials therefore find application for filtration membranes (Desai et al. 2009), in drug delivery (M. Huang et al. 2005; Fröhlich 2012; Honary \& Zahir 2013a; Honary \& Zahir 2013b) and as sensors (Liu et al. 2004; Martins et al. 2012). Additionally, the amount of accessible amine groups was found to determine the physical and biological characteristics of the membranes (Dash et al. 2011). Accessible amine groups affect properties of the following: (1) the electronic surface charge distribution (Lavertu et al. 2006; M. Huang et al. 2005), (2) the hydrophilic/hydrophobic nature (Zhu et al. 2008), (3) the antimicrobial activity and metal chelating capacity (Desai et al. 2009), (4) the water absorption and swelling properties (Gámiz-González et al. 2015; Zhou, Kong, X. J. Cheng, et al. 2014), (5) the hemo-compatibility (Fischer et al. 2003; Zhou, Kong, X. Cheng, et al. 2014), (6) the cell adhesion, growth and differentiation (Dillon et al. 2000; Chatelet et al. 2001; Tchemtchoua et al. 2011; Bertoncelj et al. 2014; Ko et al. 2016).

To tailor physical and biological characteristics of chitosan non-wovens towards specific needs, various parameters have to be controlled closely to get the necessary surface chemistry and fiber morphology. These parameters are governed by (1) the polymer(s) being spun (including their chemistry (Desai et al. 2008; Klossner et al. 2008; Li \& Hsieh 2006), their molecular weight (Desai et al. 2008; Zhang et al. 2008; Zhang et al. 2009), their mixing ratio (Duan et al. 2004; Zhang et al. 2009) and their ionic nature), (2) the solvent system used (i.e. its type, its mixing ratio (Duan et al. 2004; Pakravan et al. 2012a; Jacobs et al. 2011; Kriegel et al. 2009), its polymer concentration and added salts or surfactants), (3) the processing parameters and (4) the ambient conditions (Gönen et al. 2016). The identification of the parameters that are significant to the spinning process as well as the optimization of the system to- 
wards a specific response, are conventionally achieved using a systematic approach where only a single factor is changed at any one time while all others are kept constant (Duan et al. 2004; Bizarria et al. 2014; Klossner et al. 2008; Kazemi Pilehrood et al. 2014). Such an approach, however, fails to take the interactive effects between the different factors into account and generally results in only one 'apparent' set of optimal conditions (Schubert et al. 2012). Such shortcomings can be solved by applying response surface methodology (RSM), a powerful statistical tool fitting an empirical model to experimental data that describes the relationship between a group of controllable input variables and one or many observable responses. RSM is widely used to optimize processes or products and allows the simultaneous investigation of individual process parameters and their interactions with each other (Gönen et al. 2016; Meyers et al. 2016; Zhou et al. 2013). Hence, RSM has been used extensively on various chitosan blends to analyze the effects of electrospinning parameters on the fiber diameter and to produce fibrous mats with targeted fiber diameter (Agarwal et al. 2012; Gholipour et al. 2010; Thirugnanasambandham \& Sivakumar 2016; Askari et al. 2014; Jacobs et al. 2011).

The aim of this study was to develop a model able to predict the amount of nitrogen surface content in chitosan/PEO blends as a function of the solution parameters used. Emphasis was given to five solution parameters: the molecular weight of the polymers, the blending ratio of chitosan/PEO (C/P), the degree of deacetylation (DD) of the chitosan, and the concentration of acetic acid in the water-based solvent. In order to identify the most significant parameters and their interactive effects, a response surface methodology (RSM) was used which is based on a three level five variables Box-Behnken (BB) design. The presented model should contribute to a deeper understanding of the spinning of chitosan/PEO blends and provide a tool for the production of membranes with tailored surface properties. 


\section{Materials and Methods}

2.1 Materials:

All chemicals were of analytical grade and used as received unless stated otherwise. Chitosan (Chitoscience, $<30,<70$ and $<150 \mathrm{mPa}$ s, $1 \%$ in $1 \%$ acetic acid; degree of deacetylation $75-95 \%$, as stated by the manufacturer cf. table S1) was purchased from HMC (Halle (Saale), Germany). Poly(ethylene oxide) with molecular weights of $200 \mathrm{kDa}, 600 \mathrm{kDa}$ and $1 \mathrm{MDa}$, acetic acid, ethanol, Dulbecco's Phosphate buffered saline (modified, without calcium chloride and magnesium chloride) and glutaraldehyde solution (GA, 50 wt \% in $\mathrm{H}_{2} \mathrm{O}$ ) were obtained from Sigma-Aldrich (Buchs, Switzerland).

\subsection{Preparation and characterization of polymer solutions:}

All solutions were prepared using ultrapure water (MilliQ). Concentrations of polymer solutions were given as wt/wt \%, unless stated otherwise. Chitosan-PEO solutions with total polymer concentrations of $4.0 \%$ were prepared by dissolving the chitosan and PEO of different molecular weights in 70,80 or $90 \%$ acetic acid. As demonstrated by Rinaudo (Rinaudo et al. 1999) the viscosity and conductivity of chitosan in solution are highly dependent on the amount of acetic acid being used. Although the mixtures of chitosan/PEO under investigation were soluble in acetic acid at lower concentrations their high conductivity negatively influenced the electrospinning process and prevented homogenous fiber formation. Ratio of chitosan to PEO were chosen as 70:30, 82.5:17.5 and 95:5, being sufficient for PEO to elicit a positive effect on the spinnability, while at the same preventing a complete masking of the desired amine functionalities of chitosan. Solutions were placed on a tabletop orbital shaker (3020, GFL, Burgwedel, Germany) at ambient conditions until complete dissolution to obtain homogeneous mixtures. Solutions were characterized and spun within $48 \mathrm{~h}$ after preparation to avoid aging effects and prevent polymer degradation (Klossner et al. 2008; Mironov et al. 2007). Zero shear rate viscosity (n0) of the solutions was determined using a Physica MCR 300 rheometer by Anton Paar (Buchs, Switzerland) with cone-plate geometry. Flow curves were measured with shear rates varying from 0.01 to $500 \mathrm{~s}^{-1}$. To avoid and eliminate any aging effects of the solutions a preshearing of $50 \mathrm{~s}^{-1}$ was applied for $30 \mathrm{~s}$ prior to measurement. Solutions were equilibrated at $25{ }^{\circ} \mathrm{C}$ prior to measurements. The results given are the averaged values of triplicates. The conductivity of the spinning solutions was measured at ambient temperatures using a 660 conductometer from Metrohm (Herisau, Switzerland) equipped 
with a Pt 100 dip-type conductometric cell $\left(c=0.83 \mathrm{~cm}^{-1}\right)$. Reported values are averages of triplicates.

\subsection{Electrospinning:}

The electrospinning setup consisted of an infusion pump (World precision instruments, Sarasota, FL, USA) equipped with a $3 \mathrm{ml}$ plastic syringe (Henke-Sass Wolf, Tuttlingen, Germany) containing the spinning solution. Spinning parameters were kept constant applying a flow rate of $10 \mu \mathrm{l} / \mathrm{min}$, a tip to collector distance of $12 \mathrm{~cm}$ and a voltage of $12 \mathrm{kV} /-4 \mathrm{kV}$. A $21 \mathrm{G} \times 7 / 8$ " blunt needle (Braun, Melsungen, Germany) served as the positive electrode while fibers were assembled on a tinfoil covered custom made rotating drum $(\varnothing 10 \mathrm{~cm}$, axis $15 \mathrm{~cm}$, rotational speed $5 \mathrm{~Hz}$ ) serving as the counter electrode. The voltage was generated by two voltage supply sources (AIP Wild AG, Oberglatt, Switzerland). The electrical field strength was controlled using LabView software (National Instruments, Austin, TX, USA). The whole equipment was placed in a grounded Faraday cage being located in a mobile fume hood (Captair Flex XLS 392, Erlab, Val de Reuil Cedex, France). All spinning experiments were conducted in a climatic chamber providing controlled environmental conditions ( $30 \pm 5 \% \mathrm{RH}$ and $22 \pm 0.5^{\circ} \mathrm{C}$ ).

\subsection{Crosslinking:}

Postprocessing was necessary to stabilize the as spun chitosan-PEO fibers for further experiments and applications taking place often in aqueous environments. This was either achieved by chemical crosslinking by glutaraldehyde vapor (Schiffman \& Schauer 2007) or by immersion in EtOH acting as a syneresis agent (Y. Huang et al. 2005; Chen et al. 2006; Mengistu Lemma et al. 2016). Vapor crosslinking by glutaraldehyde took place in a desiccator (volume $1 \mathrm{~L}$ ) containing $4 \mathrm{ml}$ of aqueous GA solution (50 wt \%). After $4 \mathrm{~h}$ membranes were removed and dried in a vacuum oven at 40 ${ }^{\circ} \mathrm{C}$ in order to remove any unreacted GA. For crosslinking by $\mathrm{EtOH}$ the membranes were immersed for $24 \mathrm{~h}$ and subsequently dried and washed in MilliQ water for another $24 \mathrm{~h}$.

\subsection{Fiber and membrane characterization:}

2.5.1. Scanning electron microscopy (SEM): 
SEM images of fibers were recorded on a Hitachi S-4800 (Hitachi High-Technologies, Canada) at an accelerating voltage of $2 \mathrm{kV}$ and current flow of $10 \mu \mathrm{A}$. Membranes were mounted onto stubs with double sided adhesive tape and sputter coated with 5 $\mathrm{nm}$ of gold/palladium prior to imaging (Polaron Equipment, SEM coating Unit E5100, Kontron AG, Switzerland) to reduce electron charging effects. Mean fiber diameter were determined from 100 individual measurements taken within representative microscopic fields using ImageJ software $1.47 \mathrm{v}$ (National Institutes of Health, USA). 


\subsubsection{X-ray photoelectron spectroscopy (XPS):}

Surface compositions were characterized by XPS measurements, performed on a $\mathrm{PHI} 5000$ VersaProbe II instrument (USA) with a monochromatic AlKa X-ray source. Energy resolution of the spectrometer was set to $0.8 \mathrm{eV} / \mathrm{step}$ at a pass-energy of $187.85 \mathrm{eV}$ for survey scans and $0.125 \mathrm{eV} / \mathrm{step}$ and $29.35 \mathrm{eV}$ pass-energy for high resolution region scans, respectively. Carbon $1 \mathrm{~s}$ at $284.5 \mathrm{eV}$ was used as a calibration reference to correct for charge effects. Elemental compositions were determined using instrument dependent atom sensitivity factors. The photoelectron-transitions $\mathrm{C} 1 \mathrm{~s}, \mathrm{O} 1 \mathrm{~s}$ and $\mathrm{N} 1 \mathrm{~s}$ were selected to determine the elemental concentrations and the chemical shifts within the region scans. Data analysis was performed by use of CasaXP software (Casa Software Ltd, United Kingdom).

\subsubsection{Fourier transform infrared spectroscopy (FTIR):}

FTIR spectra were recorded on a Bruker Tensor 27 equipped with a Pike GladiATR (Bruker Optics $\mathrm{GmbH}$, Fällanden, Switzerland) accessory. Measurements are composed of 32 scans recorded at wave numbers between 4000 and $500 \mathrm{~cm}^{-1}$ at a resolution of $4 \mathrm{~cm}^{-1}$. Spectra were analyzed using OPUS (v.7) software.

\subsubsection{Elemental analysis (EA):}

EA was performed in duplicates by Mikroanalytisches Labor Pascher (Remagen, Germany) with the uncertainty of measurement being within $\pm 0.2 \%$ for the elements carbon, hydrogen and nitrogen and within $\pm 0.5 \%$ for oxygen respectively.

\subsubsection{Transmission electron microscopy (TEM):}

In order to investigate the polymer phase distribution within the electrospun chitosan-PEO fibers, membranes $\left(5 \mathrm{~mm}^{2}\right)$ were embedded and subsequently cut into ultra-thin sections $(\sim 100 \mathrm{~nm})$ means of an Ultracut E microtome (Leica Biosystems, Wetzlar, Germany). The embedding took place in a vacuum chamber for $1 \mathrm{~h}$ using freshly prepared epoxy medium (Sigma-Aldrich, Buchs, Switzerland) supplemented with the epoxy embedding medium hardener methyl nadic anhydride (MNA) (34 wt/wt \%) and dodecenylsuccinic anhydride (DDSA) (17 wt/wt \%) respectively as well as $0.02 \mathrm{wt} / \mathrm{wt} \%$ of the epoxy embedding medium accelerator 2,4,6-tris(dimethylaminomethyl)phenol (DMP-30). To complete polymerization the 
embedded membranes were placed on a silicone mat and left to rest for 3 days at $30{ }^{\circ} \mathrm{C}$ before being trimmed and coated with $\mathrm{Pt}$. In a next step the membranes were placed in a mold and embedded with another layer of epoxy embedding medium. Finally the resulting polymer block was cut into thin sections, positioned on 400 mesh copper grids and examined with a FEI/Philips CM 12 TEM (FEI Company, Hillsboro, Oregon, United States).

\subsubsection{Zeta potential measurements:}

The net surface charge of the membranes was assessed by streaming potential measurements using a SurPASS Electrokinetic Analyzer (Anton Paar, Austria). Samples with dimensions of $20 \times 10 \mathrm{~mm}^{2}$ were mounted in an adjustable gap cell (gap height was $100 \mu \mathrm{m} \pm 20 \mu \mathrm{m}$ ) with double-sided adhesive tape. Prior to the measurements, samples were washed and rinsed with $1 \mathrm{mM} \mathrm{KCl}$ electrolyte solution and a flow check was performed to ensure a linear correlation between pressure and flow. The streaming potential was measured during a pressure ramp from 0 to 200 mbar and the zeta potential was calculated from the slope by the operating software (Attract 2.1, Anton Par) according to the Fairbrother-Mastin model. Results are given as average and standard deviation obtained from four individual pressure ramps.

\subsection{Design of experiments:}

Electrospinning experiments were designed (table S2) to investigate the influence of solutions parameters on the $\mathrm{N}$ atomic surface nitrogen content applying a Box Behnken design (BB). All factors are studied in three levels $(-1,0,1)$. A second-order polynomial model was defined to fit the response:

$Y=\beta_{0}+\sum_{i=1}^{k} \beta_{i} x_{i}+\sum_{i=1}^{k} \beta_{i i} x_{i}^{2}+\sum_{i=1}^{k} \sum_{j=i+1}^{k} \beta_{i j} x_{i} x_{j}+\varepsilon$

where $\mathrm{Y}$ is response ( $\mathrm{N}$ atomic surface content), $\beta_{0}$ the off-set term called intercept , $\beta_{\mathrm{i}}, \beta_{\mathrm{ii}}, \beta_{\mathrm{ij}}$ linear coefficient, second order-interaction coefficient and quadratic coefficient respectively and $x_{i}$ are independent variables related to the factors while $\varepsilon$ is the error of the model (Ghani et al. 2014; Agarwal et al. 2012; Schubert et al. 2012). The relative importance of each term in the model was determined from t-values and associated $p$-values. Coefficients with $p$-values less than 0.05 were considered statistically significant. 
The 5 independent variables $\left(\mathrm{X}_{\mathrm{i}}\right)$ used in this study included the degree of deacetylation (\%), the viscosity of chitosan ( $\left.\mathrm{mPa}^{*} \mathrm{~s}\right)$, the molecular weight of PEO (kDa), the concentration of acetic acid in the solvent (wt \%) and the ratio of chitosan/PEO (wt/wt $\%$ ) are coded (table 1) according to the following equation:

$X=\frac{x-\left[x_{\max }+x_{\min }\right] / 2}{\left[x_{\max }-x_{\min }\right] / 2}$

where $x$ is the natural variable, $X$ is the coded variable and $x_{\max }$ and $x_{\min }$ are the maximum and minimum values of the natural variable.

To assess the fitting and predictive accuracy of the model, a graphical comparison was performed and additionally the datasets were mathematically evaluated by calculating the following evaluation criteria: coefficient of determination $\left(R^{2}\right)$ (Box \& Draper 1987), root-mean-squared error (RMSE) (Carrasco et al. 2006), mean relative percentage error (MRPE), mean absolute percentage error (MAPE) (Jeyamkonda et al. 2001), and the proportion of the relative error ( $p R E)$ (Oscar 2005). Furthermore, for validating the model, three additional external experiments within the design space but not employed in the model generation were carried out (table S3). The model development by regression analysis and the model evaluation were performed with Matlab® Software (Version R2016a; MathWorks). 


\section{Results and Discussion}

\subsection{Solution properties:}

The chain entanglement within spinning solutions is a result of the polymer concentration as well as the molecular weight of the polymers being used and therefore linked to the viscosity of the spinning solutions (Shenoy et al. 2005; Rieger et al. 2016). To better understand how the physical properties of the spinning solution influence fiber morphology, the rheological behavior (table S4/S5, figure S1) of the spinning solutions was examined along with their electrical conductivity (table S4/S5), another factor known to impact the electrospinning process (Pakravan et al. 2011; Uyar \& Besenbacher 2008). For all solutions a Newtonian flow behavior was observed in the low shear stress range with a well-developed plateau region and a nonNewtonian behavior at high shear rates with shear thinning behavior (figure S1). Of the five solution parameters under investigation the molecular weight of chitosan turned out to be the most significant for solution viscosity (table S6), while solution conductivity was mostly dependent on the percentage of acetic acid (table S7). 


\subsection{Fiber morphology:}

For the chosen process parameters all 41 investigated solutions (table S2) were successfully spun into fibers with nanoscaled diameter. Fiber morphologies were found to be homogenous except for solutions prepared using the low molecular weight PEO $(200 \mathrm{kDa})$ which resulted in beaded nonwovens. As molecular weight or rather chain length have a strong influence on chain entanglement, it seems that for those spinning solutions the critical chain entanglement concentrations $\left(\mathrm{C}_{\mathrm{e}}\right)$ were not reached to ensure a stable jet formation (Shenoy et al. 2005; Rieger et al. 2016). Only considering membranes exhibiting homogenous, non-beaded morphologies, mean fiber diameters for the electrospun membranes were found to be between $46 \mathrm{~nm}$ and 616 $\mathrm{nm}$ (figure 1, table S4). Importantly, blends with a high chitosan weight content of up to $95 \%$ were spun successfully, showing a decrease in the mean fiber diameter as a result to an increase in the percentage of chitosan in the spinning solution. A similar but much stronger effect on fiber diameter was found when lowering the amount of acetic acid in solution from 90 to $70 \mathrm{wt} \%$. In both cases, the changes raised the solution conductivity (table S5) while the effects on viscosity were negligible, resulting in a higher stretching rate of the polymer jet in the electric field (Pakravan et al. 2011). Increasing the molecular weight of PEO, however, increases solution viscosity without major changes in conductivity (table S5), resulting in less drawing during electrospinning and in a larger fiber diameter (table S4). The degree of deacetylation as along with the viscosity of the chitosan, exhibited non-linear effects on fiber diameter with local maxima observed for chitosans, respectively exhibiting a DD of $85 \%$ and a viscosity of $50 \mathrm{mPa}{ }^{*} \mathrm{~s}$. For chitosan, there is a complex interplay between chain length and DD responsible for the degree of hydrophobic, steric and electrostatic interaction which govern the hydrodynamic radii and the charge density on the chitosan chains (Anthonsen et al. 1993; Lamarque et al. 2005; Mucha 1998; Schatz et al. 2003). This results in a local maxima of fiber diameter observed for chitosan with a viscosity of 50 mPa*s and a DD of $85 \%$, respectively. No direct correlation with the resultant fiber diameter was found for both solution conductivity or viscosity. Although, in general the fiber diameter is thought to shrink with increasing solution viscosity and higher conductivity as both variables should result in higher drawing rates, subsequently resulting in thinner fibers (Pakravan et al. 2011). However the observation that output parameters of chitosan-PEO blended systems behave contradictory to the generalized doctrine was previously made by other groups (Zhang et al. 2009; Klossner et al. 2008) and attributed to the polyelectrolyte nature of chitosan. Furthermore, Helgeson (Helgeson et al. 2008) pointed out the shortcomings of fiber diameter predictions solely based on single parameters such as zero-shear viscosity while not taking into account the apparent role of extensional rheology of the electrospinning process. 
Modeling the accessible nitrogen surface concentration:

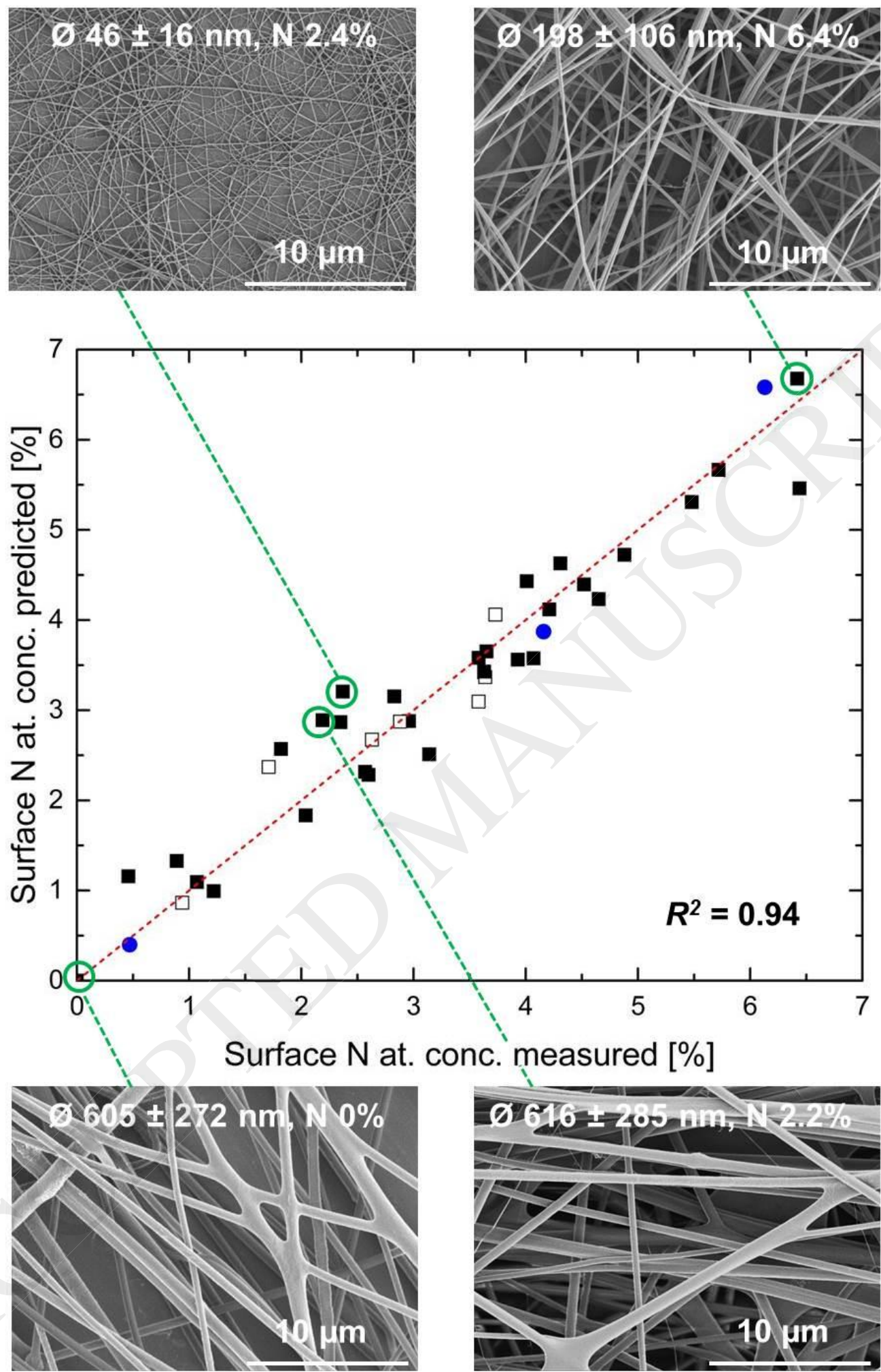

Figure 1: Nitrogen content measured vs. predicted for the 41 samples spun. SEM pictures show the fiber morphologies of the samples with the highest and lowest nitrogen surface composition, as well as the biggest and smallest homogenous fibers respectively. Black squares stand for homogenous fibers, empty squares show beaded morphologies. Blue circles were chosen for more detailed chemical analysis in the following sections. 


\subsection{Modeling:}

The atomic nitrogen content at the surface measured by XPS analysis varied between 0 and $6.4 \%$ (figure 1, table S4) in comparison to $7.5 \%$, as measured for a neat chitosan film. Evaluation of the reliability and the robustness of the model were based on both graphical plot (figure 1) and evaluation indices. Besides the high coefficient value of $R^{2}=0.94$, the indices RMSE (0.380) (Baranyi \& Roberts 1995; Fernández \& Peck 1997), MRPE (-2.787), MAPE (15.59) (Jeyamkonda et al. 2001; Hajmeer et al. 1997) and pRE (0.826) (Oscar 2005) were in good agreement with literature values reported for validated predictive models, thus confirming the excellent prediction accuracy and generalization ability of the RS model we applied. Moreover, three additional experiments being within the design space but not employed in the model generation were performed (table S3). The predicted values were found to be in close agreement with the experimental findings, confirming the model to be valid. Two linear terms (chitosan molecular weight and PEO/chitosan mixing ratio), two quadratic terms (PEO molecular weight and PEO/chitosan mixing ratio) and three interaction terms (table 2, figure S2) were identified to be statistically significant regarding the nitrogen atomic surface content.

Coefficient analysis (table 2) revealed the chitosan/PEO ratio to be the most influential parameter for the $\mathrm{N}$ atomic surface concentration. The nitrogen surface concentration was found to rise linearly with increasing chitosan ratio in the spinning solution. When applying a ratio of chitosan/PEO of 95/5 wt/wt \%, the surface nitrogen content reached levels that were in line with the theoretical bulk composition. When decreasing the chitosan concentration in the spinning solution a strong leverage effect on the surface nitrogen content was observed and fiber characteristics began to resemble that of pure PEO fibers as confirmed by FTIR (figure S3) and XPS (figure 3) measurements.

Elemental analyses were undertaken on fibrous mats (table 3) to investigate the discrepancy between theoretical and measured (XPS) atomic surface nitrogen composition. By doing so, the possibility of this discrepancy being the effect of a phase separation in the spinning solution changing the polymer ratio among the aliquots taken for electrospinning could be ruled out. Elemental analysis confirmed that chitosan was still present within the fibrous mats at levels being identical to the ones used for the initial spinning solutions. This indicates that a change in the chitosan PEO ratio 
from $95 / 5$ to $70 / 30$ alters the behavior of the solution in the spinning process. The result is an alteration in fiber morphology, which leads to a decrease in accessible amine groups at the fiber surface. Zhang (Zhang et al. 2009) suggested that a shift in the phase diagram between stable and metastable region might be responsible for the divergent behavior observed for different chitosan/PEO blending ratio during the electrospinning process. Also, a similar explanation for the occurrence of a PEO ratio driven fiber morphology was proposed by Li (Li et al. 2014).

The molecular weight of chitosan turned out to be another significant parameter. An increase in molecular weight did result in a linear decrease in nitrogen surface concentration. This is thought to be the result of a decreased mobility due to a higher chain length and a stronger entanglement. Furthermore the higher molecular weight resulted in a simultaneous increase in viscosity, which may have reduced solvent evaporation. A lower evaporation rate could lead to a slow polymer solidification allowing for a stronger phase separation in the process of electrospinning, resulting in a more pronounced polymer gradient within the resulting fibers (Wang et al. 2015). For shorter chitosan chains on the other hand migration in the electric field during the electrospinning process is facilitated leading to an enrichment of amine groups at the fiber surface (Zhang et al. 2009). Additionally, in systems with two components the attempt to attain a state of minimum energy dissipation will favor the migration of the constituent with a lower viscosity towards locations exhibiting the highest shear rates which are located at the needle walls (Chen et al. 2010).

The molecular weight of PEO influences the nitrogen surface content in a quadratic manner with maximum nitrogen surface contents observed for medium molecular weight PEO. Molecules of lower molecular weight have a higher mobility which has been shown to facilitate phase separation and the formation of core-sheath structures (Wei et al. 2006). In the case of PEO, which has a low molecular weight $(200 \mathrm{kDa})$, the low atomic nitrogen surface content might therefore be explained by the formation of a PEO gradient because of its facile migration. Increasing PEO molecular weight lowers its mobility and impedes phase separation which raises the nitrogen surface content. For PEO with a medium molecular weight the elemental surface composition and the bulk composition were in good agreement, thus confirming a continuous phase morphology. For high molecular weight PEO (1 MDa), the decrease in the amount of atomic nitrogen surface content was considered to be the result of a PEO shell formation. Due to its lower boiling point in comparison to acetic acid $\left(118^{\circ} \mathrm{C}\right)$, water evaporates faster than acetic acid at the surface of the electrospinning jet. As water is only a solvent for PEO, its evaporation results in the formation of a skin made up of nearly pure PEO at the fiber-air interface (Chen et al. 
2010; Wei et al. 2006; Jiang et al. 2013; Guenthner et al. 2006). PEO is prone to solidify upon solvent evaporation, as its high molecular weight reduces its solubility (Sakellariou et al. 1994; Miller-Chou \& Koenig 2003) as well as its ability to migrate towards the fiber core. Furthermore, PEO is less soluble in the remaining acetic acid in comparison to chitosan resulting in the formation of a polymer gradient within the fiber (Pakravan et al. 2012b). Moreover a molecular weight driven structure formation of PEO e-spun polymer blends has previously been reported by others (Fortunato et al. 2014). The other solution parameters including the percentage of acetic acid in the solvent along with the degree of deacetylation were both found to have no significant effect on the amount of nitrogen being present at the fiber surface. 


\subsection{SEM:}

After identifying the chitosan/PEO ratio to be the most influential factor for surface nitrogen content out of the 41 samples used in model generation, three samples were chosen in order to demonstrate how tailoring the nitrogen content can change the chemical and physical properties of the chitosan membranes. These 3 samples varied only in their chitosan/PEO ratio but were otherwise identical regarding the rest of the solution parameters. They exhibited homogenous fiber morphologies with largest mean fiber diameter for the blend containing the highest percentage of PEO while the smallest fibers were obtained for a chitosan/PEO ratio of 82.5/17.5 wt/wt \% (figure 2). TEM images of whole fibers and fiber cross-sections (figure S4) did not reveal the presence of a core-shell structure. However, a certain degree of phase separation was discernible as indicated by the inhomogeneous grey scale pattern of the cross sections, which is underpinned by the XPS surface elemental composition results revealing an enrichment of PEO at fibers surfaces.

\section{Ratio chitosan/PEO}

$70 / 30$
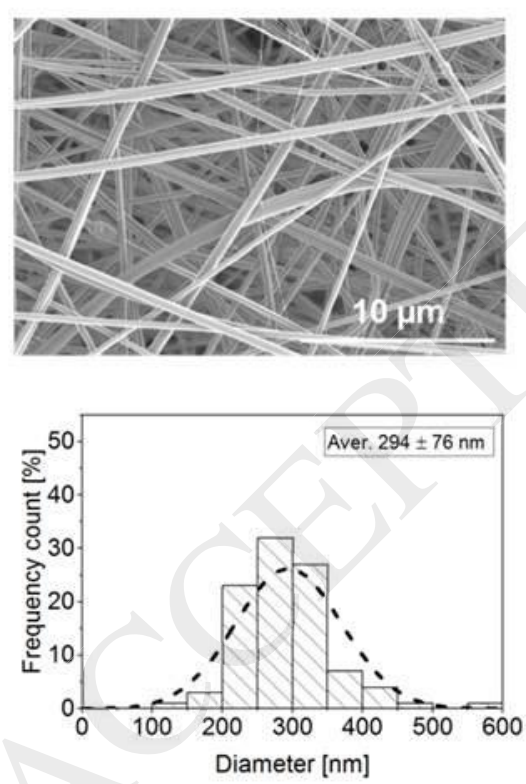

$82.5 / 17.5$
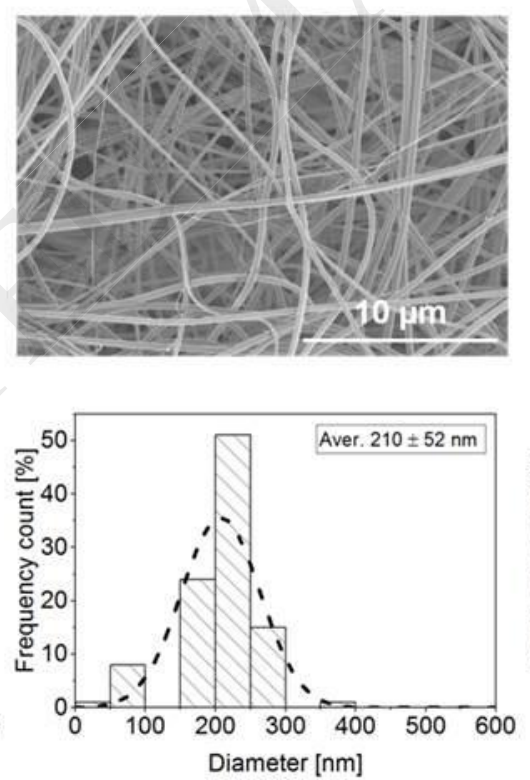

$95 / 5$
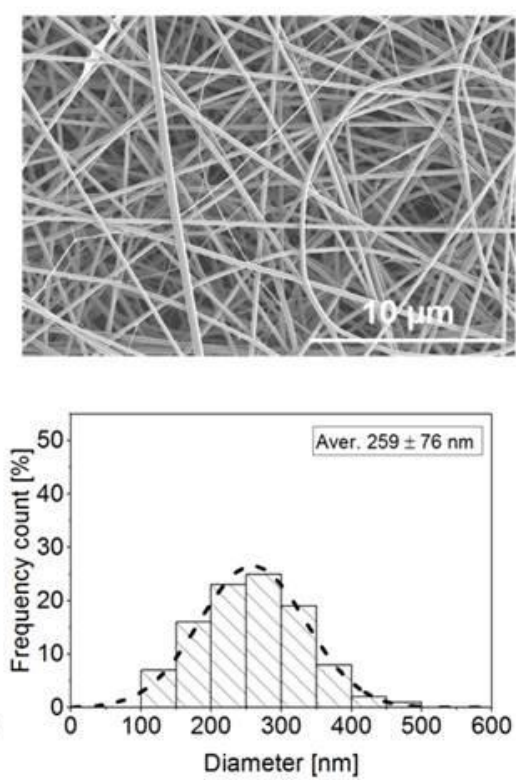

Figure 2: The influence of mixing ratio on fiber morphology and diameter as revealed by SEM.

\subsection{Spectroscopic analysis:}


Electrospun fibrous mats were subject to FTIR and XPS spectroscopic analyses to determine their chemical characteristics. The FTIR spectra of the blends (figure S3) clearly show that the blended fibrous mats exhibit chemical characteristics of both the polymers present in the spinning solution, thus indicating only minor interactions between the two components in the solid state due to no shifts in the signal wavenumbers being found. The intensity of the different signals is, however, strongly dependent on the mixing ratio. XPS spectra revealed changes in the characteristic line shapes of the carbon $\mathrm{C} 1 \mathrm{~s}$ (figure 3, detailed interpretation appendix) environment which were dependent on the chitosan PEO ratio of the spinning solution. For a chitosan/PEO ratio of $70 / 30 \mathrm{wt} / \mathrm{wt} \%$, a sharp peak was identified, resembling the spectra of pure PEO. With an increase in the amount of chitosan the signal started to broaden, up to a ratio of chitosan/PEO of $95 / 5 \mathrm{wt} / \mathrm{wt} \%$ where the line shape of the C1s environment approaches that of neat chitosan. Furthermore, the N1s peak only changed in size and not in shape when raising the amount of chitosan in the blends. With a decreasing chitosan content in the blend, the deviation between atomic surface and bulk composition became more pronounced (cf. table 3 ) with an almost complete depletion of the N1s for a chitosan/PEO ratio of $70 / 30 \mathrm{wt} / \mathrm{wt} \%$.
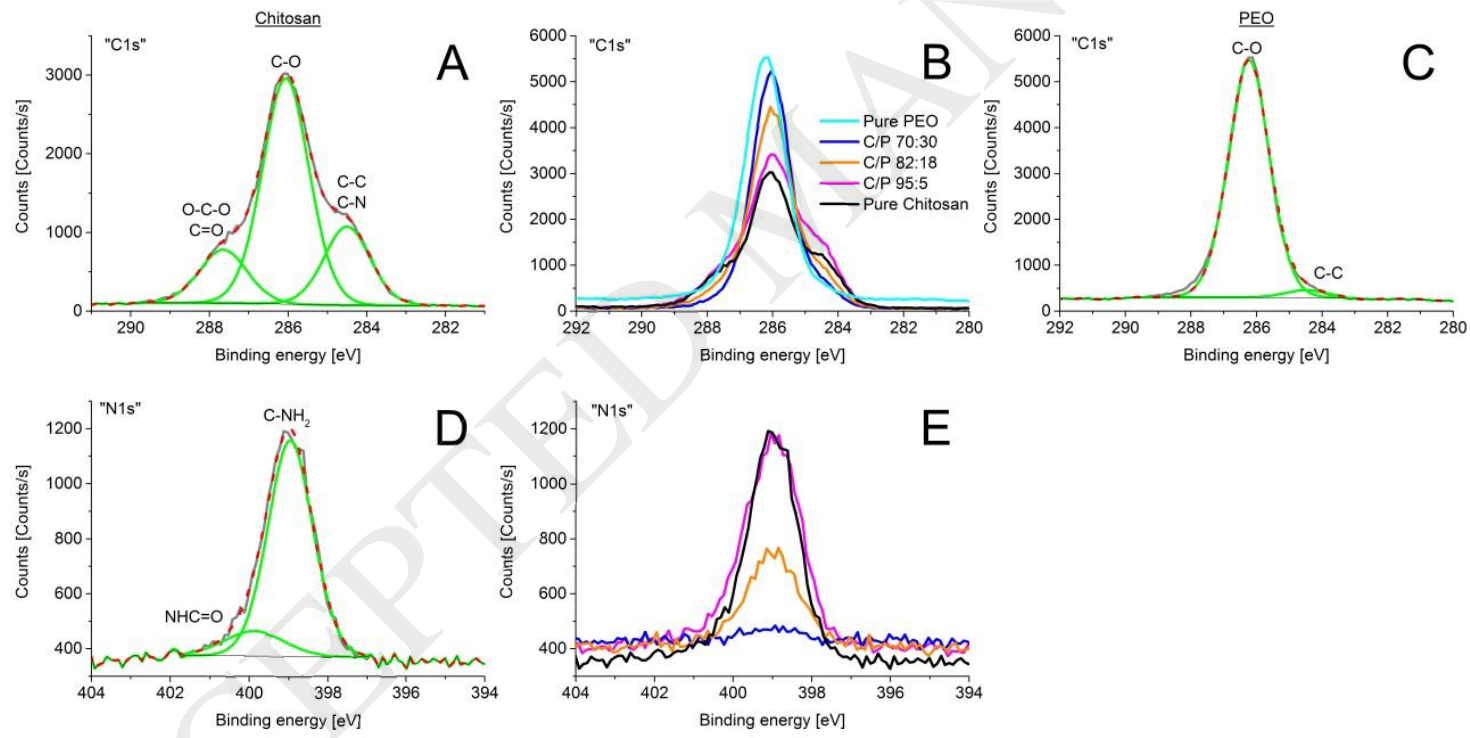

Figure 3: High resolution C1s and N1s XPS transitions with fittings of the two starting materials pure chitosan (A and D) and pure PEO (C). B and E are elemental scans of the carbon and nitrogen environment obtained for the blended fibers. 
In order to monitor the changes in physical surface properties associated with alteration of the atomic nitrogen surface composition the surface zeta potential of the 3 selected membranes were measured. Spun membranes were soluble in aqueous environment and firstly had to be chemically stabilized by means of glutaraldehyde (GA) or EtOH. Stabilization by means of EtOH generally lead to an increase of nitrogen atomic surface concentration in comparison to the initial non-crosslinked sample. This increase in available nitrogen groups at the surface in the course of crosslinking is thought to be caused by a partial dissolution of $\mathrm{PEO}$ by $\mathrm{EtOH}$. We suggest that the stabilizing effect of the EtOH treatment for PEO results from a loss of residual water within the polymer structure which increases crystallinity and mechanical strength, respectively as observed for the pure PEO (Ho et al. 2006; Zhang et al. 2012) and PVA (Yao et al., 2003).

GA crosslinking in contrast reduced the amount of available nitrogen groups at the fiber surface especially when starting from membranes with a high nitrogen atomic surface content. This observation coincided with the proposed reaction mechanism via the Schiff's base mechanism supposedly followed by a formation of a GA multilayer burying nitrogen groups. The nitrogen atomic surface content is a measure for the amount of amine groups and upon fitting of the N1s signals the amount of accessible amine functionalities. These amine groups, exhibiting a pKa value in the range of $6.17-6.51$ (Wang et al. 2006), are protonated in acidic environment resulting in an increase in charge density which leads to an increase in zeta potential as previously demonstrated (Kiang et al. 2004; Lavertu et al. 2006). The crosslinked membranes exhibited nitrogen atomic surface contents between 1.1 and $6.5 \%$ which resulted in measured zeta potential between -1.72 and $16.24 \mathrm{mV}$ (cf. figure 4). The logarithmic relationship between nitrogen atomic surface content and zeta potential observed in this study is in good agreement with findings made previously e.g. for chitosan nanoparticle systems (Kiang et al. 2004; Lavertu et al. 2006). 

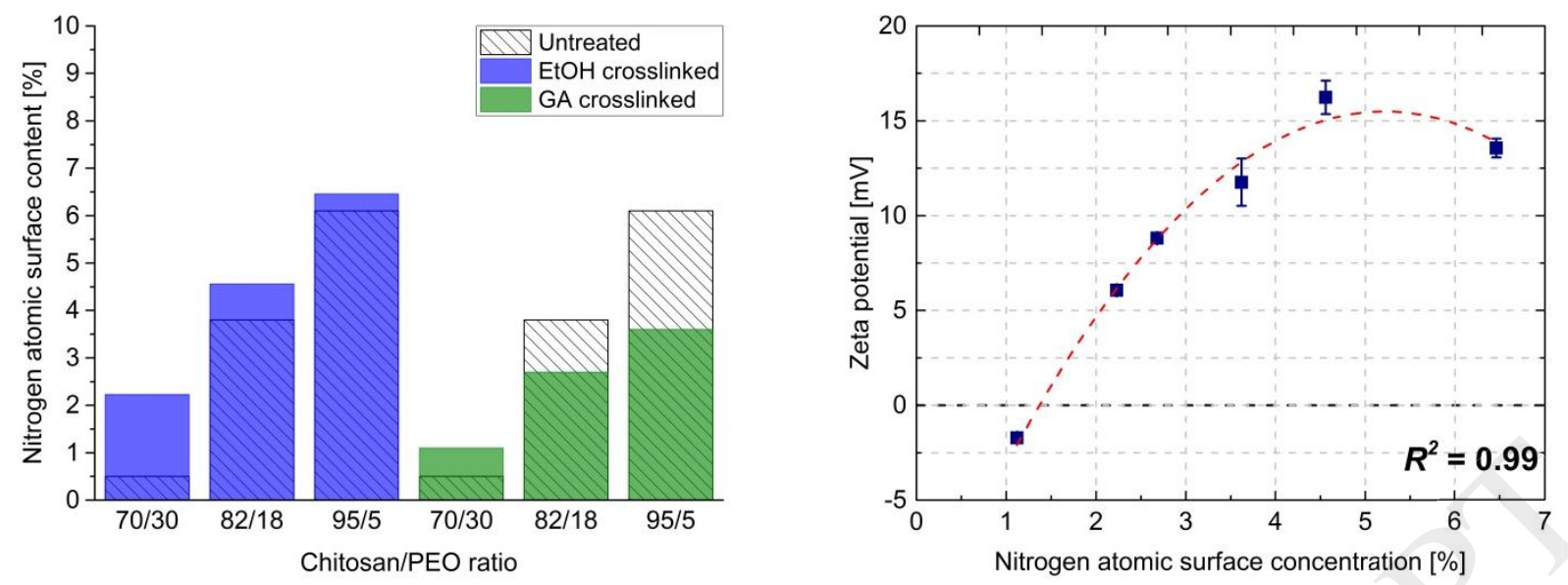

Figure 4: The effect of different stabilizing agents on the nitrogen atomic surface content of membranes exhibiting various chitosan/PEO ratio (left) and the correlation between $\mathrm{N}$ atomic concentration and zeta potential for the stabilized membranes. 


\section{Conclusion}

This study presents a highly accurate response surface modeling approach based on a three level, five variable BB design to correlate solution parameters with the amount of accessible nitrogen groups at the surface of electrospun chitosan/PEO blended fibers produced in a non-coaxial setup. The model revealed how the blending ratio, the degree of deacetylation of chitosan, the percentage of acetic acid in solution and the molecular weight of chitosan as well as PEO influence the amount of nitrogen atomic surface concentration and how those parameters interfere with each other. The results identified the blending ratio to be the most significant parameter for a high nitrogen surface content while a negative correlation was found with increasing chitosan and PEO molecular weight. SEM images confirmed that, under the experimental conditions and within the design space applied, homogenous fibers with similar morphology were formed, irrespective of the solution composition being used. XPS, FTIR and EA measurements, however, clearly demonstrated a strong discrepancy between atomic surface nitrogen content and bulk composition especially for lower chitosan/PEO ratio which is thought to be the result of molecular weight and electronic repulsion driven phase separation behavior. The potential of the predictive RSM to tailor physical and biological characteristics of chitosan non-wovens has been demonstrated using the example of the membrane zeta potential. The amount of nitrogen atomic surface content has been shown to positively correlate with the measured zeta potential, which is known to be linked with the cell adhesion and proliferation (Chen et al. 2011; Jeong et al. 2013; Croisier et al. 2014) as well as with the antimicrobial activity (Chang et al. 2015; Ceylan et al. 2017) exhibited by the membrane. Although the model is applicable only under the experimental conditions inside the design space, the potential of RSM in modeling the surface properties of chitosan fibers as an outcome of a multi-parameter process is promising and could pave a way towards rationally designed drug delivery systems or tissue engineering applications based on chitosan non-wovens. 


\section{Acknowledgements}

The authors acknowledge Anne-Greet Bittermann and Eszter Barthay from the Scientific Center for Optical and Electron Microscopy ScopeM of the Swiss Federal Institute of Technology ETHZ for their technical assistance with TEM measurements. 


\section{References}

Agarwal, P., Mishra, P.K. \& Srivastava, P., 2012. Statistical optimization of the electrospinning process for chitosan/polylactide nanofabrication using response surface methodology. Journal of Materials Science, 47(10), pp.4262-4269.

Ajalloueian, F. et al., 2014. Emulsion electrospinning as an approach to Fabricate PLGA/chitosan nanofibers for biomedical applications. BioMed Research International, 2014.

Aliabadi, M. et al., 2013. Electrospun nanofiber membrane of PEO/Chitosan for the adsorption of nickel, cadmium, lead and copper ions from aqueous solution. Chemical Engineering Journal, 220, pp.237-243.

Anthonsen, M.W., Vårum, K.M. \& Smidsrød, O., 1993. Solution properties of chitosans: conformation and chain stiffness of chitosans with different degrees of N-acetylation. Carbohydrate Polymers, 22(3), pp.193-201.

Askari, M. et al., 2014. Fabrication of high performance chitosan/polyvinyl alcohol nanofibrous mat with controlled morphology and optimised diameter. Canadian Journal of Chemical Engineering, 92(6), pp.1008-1015.

Baranyi, J. \& Roberts, T.A., 1995. Mathematics of predictive food microbiology. International Journal of Food Microbiology, 26(2), pp.199-218.

Bertoncelj, V. et al., 2014. Development and bioevaluation of nanofibers with bloodderived growth factors for dermal wound healing. European Journal of Pharmaceutics and Biopharmaceutics, 88, pp.64-74.

Bhattarai, N. et al., 2005. Electrospun chitosan-based nanofibers and their cellular compatibility. Biomaterials, 26(31), pp.6176-6184.

Bizarria, M.T.M., D 'ávila, M.A. \& Mei, L.H.I., 2014. NON-WOVEN NANOFIBER CHITOSAN/PEO MEMBRANES OBTAINED BY ELECTROSPINNING. Brazilian Journal of Chemical Engineering, 31(1), pp.57-68. Available at: www.abeq.org.br/bjche [Accessed August 10, 2016].

Box, G.E.P. \& Draper, N.R., 1987. Empirical Model-Building and Response Surfaces, New York: Wiley. Available at: http://psycnet.apa.org/psycinfo/1987-97236-000.

Carrasco, E. et al., 2006. Predictive Model of Listeria Monocytogenes' Growth Rate Under Different Temperatures and Acids. Food Science and Technology International, 12(1), pp.47-56. Available at: http://fst.sagepub.com/cgi/doi/10.1177/1082013206062234.

Ceylan, Z., Sengor, G.F.U. \& Yilmaz, M.T., 2017. A Novel Approach to Limit Chemical Deterioration of Gilthead Sea Bream (Sparus aurata) Fillets: Coating with Electrospun Nanofibers as Characterized by Molecular, Thermal, and Microstructural Properties. Journal of Food Science, 82(5), pp.1163-1170. Available at: http://doi.wiley.com/10.1111/1750-3841.13688.

Chang, S.H. et al., 2015. pH Effects on solubility, zeta potential, and correlation between antibacterial activity and molecular weight of chitosan. Carbohydrate Polymers, 134, pp.74-81. Available at: http://dx.doi.org/10.1016/j.carbpol.2015.07.072.

Chatelet, C., Damour, O. \& Domard, a, 2001. Influence of the degree of acetylation on some biological properties of chitosan films. Biomaterials, 22(3), pp.261-268.

Chen, H. et al., 2011. Electrospun chitosan-graft-poly ( $\varepsilon$-caprolactone)/poly $(\varepsilon-$ 
caprolactone) cationic nanofibrous mats as potential scaffolds for skin tissue engineering. International Journal of Biological Macromolecules, 48(1), pp.1319. Available at: http://dx.doi.org/10.1016/j.ijbiomac.2010.09.019.

Chen, M. et al., 2010. Thermo-responsive core-sheath electrospun nanofibers from poly ( $\mathrm{N}$-isopropylacrylamide)/polycaprolactone blends. Chemistry of Materials, 22(14), pp.4214-4221.

Chen, X.G. et al., 2006. Preparation and biocompatibility of chitosan microcarriers as biomaterial. Biochemical Engineering Journal, 27(3), pp.269-274.

Cheng, F. et al., 2015. Composite chitosan/poly(ethylene oxide) electrospun nanofibrous mats as novel wound dressing matrixes for the controlled release of drugs. Journal of Applied Polymer Science, 132(24), pp.1-8.

Croce, M. et al., 2016. Synthesis and screening of $\mathrm{N}$-acyl thiolated chitosans for antibacterial applications. Carbohydrate Polymers, 151, pp.1184-1192. Available at: http://linkinghub.elsevier.com/retrieve/pii/S0144861716306774.

Croisier, F. et al., 2014. Polysaccharide-Coated PCL Nanofibers for Wound Dressing Applications. Advanced Healthcare Materials, 3(12), pp.2032-2039.

Dash, M. et al., 2011. Chitosan - A versatile semi-synthetic polymer in biomedical applications. Progress in Polymer Science (Oxford), 36(8), pp.981-1014. Available at: http://dx.doi.org/10.1016/j.progpolymsci.2011.02.001.

Desai, K. et al., 2008. Morphological and Surface Properties of Electrospun Chitosan Nanofibers Morphological and Surface Properties of Electrospun Chitosan. Biomacromolecules, 9(January), pp.1000-1006.

Desai, K. et al., 2009. Nanofibrous chitosan non-wovens for filtration applications. Polymer, 50(15), pp.3661-3669. Available at: http://dx.doi.org/10.1016/j.polymer.2009.05.058.

Dillon, G.P., Yu, X. \& Bellamkonda, R. V., 2000. The polarity and magnitude of ambient charge influences three- dimensional neurite extension from DRGs. Journal of Biomedical Materials Research, 51(3), pp.510-519.

Duan, B. et al., 2004. Electrospinning of chitosan solutions in acetic acid with poly(ethylene oxide). Journal of biomaterials science. Polymer edition, 15(6), pp.797-811.

Fernández, P.S. \& Peck, M.W., 1997. Predictive Model Describing the Effect of Prolonged Heating at 70 to $80^{\circ} \mathrm{C}$ and Incubation at Refrigeration Temperatures on Growth and Toxigenesis by Nonproteolytic Clostridium botulinum. Journal of Food Protection, 60(9), pp.1064-1071.

Fischer, D. et al., 2003. In vitro cytotoxicity testing of polycations: influence of polymer structure on cell viability and hemolysis. Biomaterials, 24, pp.1121-31. Available at: http://www.ncbi.nlm.nih.gov/pubmed/12527253\%5Cnhttp://www.sciencedirect.co $\mathrm{m} / \mathrm{science} / \mathrm{article} / \mathrm{pii} / \mathrm{S} 0142961202004453$.

Fortunato, G. et al., 2014. Molecular weight driven structure formation of PEG based e-spun polymer blend fibres. Polymer (United Kingdom), 55(14), pp.3139-3148. Available at: http://dx.doi.org/10.1016/j.polymer.2014.04.053.

Fröhlich, E., 2012. The role of surface charge in cellular uptake and cytotoxicity of medical nanoparticles. International Journal of Nanomedicine, 7, pp.5577-5591.

Gámiz-González, M.A. et al., 2015. Determining the influence of N-acetylation on water sorption in chitosan films. Carbohydrate Polymers, 133, pp.110-116. Available at: http://dx.doi.org/10.1016/j.carbpol.2015.07.020. 
Geng, X., Kwon, O.H. \& Jang, J., 2005. Electrospinning of chitosan dissolved in concentrated acetic acid solution. Biomaterials, 26(27), pp.5427-5432.

Ghani, M. et al., 2014. Fabrication of electrospun polyamide-6/chitosan nanofibrous membrane toward anionic dyes removal. Journal of Nanotechnology, 2014.

Ghitescu, R.-E. et al., 2015. Encapsulation of polyphenols into pHEMA e-spun fibers and determination of their antioxidant activities. International journal of pharmaceutics, 494(1), pp.278-87. Available at:

http://www.sciencedirect.com/science/article/pii/S0378517315301174 [Accessed July 25, 2016].

Gholipour, A., Bahrami, S.H. \& Nouri, M., 2010. Optimization of chitosanpolyvinylalcohol electrospinning process by Response Surface Methodology (RSM). E-Polymers.

Gómez-Mascaraque, L.G., Sanchez, G. \& López-Rubio, A., 2016. Impact of molecular weight on the formation of electrosprayed chitosan microcapsules as delivery vehicles for bioactive compounds. Carbohydrate Polymers, 150, pp.121-130.

Gönen, S.Ö., Erol Taygun, M. \& Kükbayrak, S., 2016. Evaluation of the factors influencing the resultant diameter of the electrospun gelatin/sodium alginate nanofibers via Box-Behnken design. Materials Science and Engineering C, 58, pp.709-723. Available at: http://dx.doi.org/10.1016/j.msec.2015.09.024.

Graf, N. et al., 2009. XPS and NEXAFS studies of aliphatic and aromatic amine species on functionalized surfaces. Surface Science, 603(18), pp.2849-2860. Available at: http://dx.doi.org/10.1016/j.susc.2009.07.029.

Guenthner, A.J. et al., 2006. Dynamics of hollow nanofiber formation during solidification subjected to solvent evaporation. Macromolecular Theory and Simulations, 15(1), pp.87-93.

Guex, A.G. et al., 2012. Fine-tuning of substrate architecture and surface chemistry promotes muscle tissue development. Acta Biomaterialia, 8(4), pp.1481-1489. Available at: http://dx.doi.org/10.1016/j.actbio.2011.12.033.

Hajmeer, M.N., Basheer, I.A. \& Najjar, Y.M., 1997. Computational neural networks for predictive microbiology II. Application to microbial growth. International Journal of Food Microbiology, 34(96), pp.51-66.

Helgeson, M.E. et al., 2008. Theory and kinematic measurements of the mechanics of stable electrospun polymer jets. Polymer, 49(12), pp.2924-2936.

Ho, D.L. et al., 2006. Unusual phase behavior in mixtures of poly(ethylene oxide) and ethyl alcohol. Journal of Polymer Science, Part B: Polymer Physics, 44(3), pp.557-564.

Honary, S. \& Zahir, F., 2013a. Effect of Zeta Potential on the Properties of Nano Drug Delivery Systems - A Review (Part 1). Tropical Journal of Pharmaceutical Research, 12(2), pp.255-264. Available at: http://www.bioline.org.br/pdf?pr13041.

Honary, S. \& Zahir, F., 2013b. Effect of Zeta Potential on the Properties of Nano Drug Delivery Systems - A Review (Part 2). Tropical Journal of Pharmaceutical Research, 12(2), pp.265-273. Available at: http://www.bioline.org.br/pdf?pr13041.

Huang, M. et al., 2005. Transfection efficiency of chitosan vectors: Effect of polymer molecular weight and degree of deacetylation. Journal of Controlled Release, 106(3), pp.391-406. 
Huang, Y. et al., 2005. In vitro characterization of chitosan-gelatin scaffolds for tissue engineering. Biomaterials, 26(36), pp.7616-7627.

Jacobs, V. et al., 2011. Optimization of Electrospinning Parameters for Chitosan Nanofibres. Current Nanoscience, 7(3), pp.396-401.

Jeong, H.G., Kim, Y.E. \& Kim, Y.J., 2013. Fabrication of poly(vinyl acetate)/polysaccharide biocomposite nanofibrous membranes for tissue engineering. Macromolecular Research, 21(11), pp.1233-1240.

Jeyamkonda, S., Jaya, D.S. \& Holle, R. a, 2001. Microbial growth modelling with artificial neural networks. International journal of food microbiology, 64(3), pp.343-354.

Jiang, Y. et al., 2013. Fabrication of core-shell nanofibers by single capillary electrospinning combined with vapor induced phase separation. New Journal of Chemistry, 37(9), p.2917. Available at: http://xlink.rsc.org/?DOI=c3nj00654a.

Kazemi Pilehrood, M. et al., 2014. Nanofibrous chitosan-polyethylene oxide engineered scaffolds: A comparative study between simulated structural characteristics and cells viability. BioMed Research International, 2014.

Khalili, S. et al., 2016. Characterization of gelatin/cellulose acetate nanofibrous scaffolds: Prediction and optimization by response surface methodology and artificial neural networks. Polymer Science Series a, 58(3), pp.399-408.

Kiang, T. et al., 2004. The effect of the degree of chitosan deacetylation on the efficiency of gene transfection. Biomaterials, 25(22), pp.5293-5301.

Kishan, A.P. et al., 2015. In situ crosslinking of electrospun gelatin for improved fiber morphology retention and tunable degradation. J. Mater. Chem. B, 3(40), pp.7930-7938. Available at:

http://pubs.rsc.org/en/content/articlehtml/2015/tb/c5tb00937e\%5Cnhttp://xlink.rsc .org/?DOI=C5TB00937E.

Klossner, R.R. et al., 2008. Correlation of Chitosan 's Rheological Properties and Its Ability to Electrospin. Biomacromolecules, 9, pp.2947-2953.

Ko, J.E. et al., 2016. Nanofiber mats composed of a chitosan-poly( D,L-lactic- coglycolic acid)-poly(ethylene oxide) blend as a postoperative anti-adhesion agent. Journal of Biomedical Materials Research Part B: Applied Biomaterials, pp.1-10. Available at: http://doi.wiley.com/10.1002/jbm.b.33726.

Kriegel, C. et al., 2009. Electrospinning of chitosan - poly ( ethylene oxide ) blend nanofibers in the presence of micellar surfactant solutions. Polymer, 50(1), pp.189-200. Available at: http://dx.doi.org/10.1016/j.polymer.2008.09.041.

Kumirska, J. et al., 2010. Application of spectroscopic methods for structural analysis of chitin and chitosan. Marine Drugs, 8(5), pp.1567-1636.

Lamarque, G. et al., 2005. Physicochemical behavior of homogeneous series of acetylated chitosans in aqueous solution: Role of various structural parameters. Biomacromolecules, 6(1), pp.131-142.

Lavertu, M. et al., 2006. High efficiency gene transfer using chitosan/DNA nanoparticles with specific combinations of molecular weight and degree of deacetylation. Biomaterials, 27(27), pp.4815-4824.

Li, J. et al., 2012. Preparation of biocompatible chitosan grafted poly(lactic acid) nanoparticles. International Journal of Biological Macromolecules, 51(3), pp.221-227. Available at: http://dx.doi.org/10.1016/j.ijbiomac.2012.05.011.

Li, L. \& Hsieh, Y. Lo, 2006. Chitosan bicomponent nanofibers and nanoporous fibers. Carbohydrate Research, 341(3), pp.374-381. 
Li, Y.-F. et al., 2014. Ultraporous interweaving electrospun microfibers from PCLPEO binary blends and their inflammatory responses. Nanoscale, 6(6), pp.33923402. Available at: http://dx.doi.org/10.1039/C3NR06197C.

Liu, H. et al., 2004. Polymeric nanowire chemical sensor. Nano Letters, 4(4), pp.671675.

Martins, P. et al., 2012. Role of nanoparticle surface charge on the nucleation of the electroactive $\beta$-poly(vinylidene fluoride) nanocomposites for sensor and actuator applications. Journal of Physical Chemistry C, 116(29), pp.15790-15794.

Matienzo, L.J. \& Winnacker, S.K., 2002. Dry processes for surface modification of a biopolymer: Chitosan. Macromolecular Materials and Engineering, 287(12), pp.871-880.

Mengistu Lemma, S., Bossard, F. \& Rinaudo, M., 2016. Preparation of Pure and Stable Chitosan Nanofibers by Electrospinning in the Presence of Poly(ethylene oxide). International Journal of Molecular Sciences, 17(12), p.1790. Available at: http://www.mdpi.com/1422-0067/17/11/1790.

Meyers, R.H., Montgomery, D.C. \& Anderson-Cook, C.M., 2016. Response Surface Methodology: Process and Product Optimization using Designed experiments 4th ed., New York: J. Wiley.

Miller-Chou, B.A. \& Koenig, J.L., 2003. A review of polymer dissolution. Progress in Polymer Science (Oxford), 28(8), pp.1223-1270.

Mironov, a. V. et al., 2007. Reasons for unstable viscous properties of chitosan solutions in acetic acid. Polymer Science Series B, 49(1-2), pp.15-17. Available at: http://link.springer.com/10.1134/S1560090407010046.

Mucha, M., 1997. Rheological characteristics of semi-dilute chitosan solutions. Macromolecular Chemistry and Physics, 198(2), pp.471-484.

Mucha, M., 1998. Rheological properties of chitosan blends with poly ( ethylene oxide ) and poly ( vinyl alcohol ) in solution. Reactive \& Functional Polymers, 38, pp.19-25.

Nisbet, D.R. et al., 2009. A review of the cellular response on electrospun nanofibers for tissue engineering. Journal of biomaterials applications, 24(1), pp.7-29. Available at: http://www.ncbi.nlm.nih.gov/pubmed/19074469.

Oscar, T.P., 2005. Validation of Lag Time and Growth Rate Models for Salmonella Typhimurium : Acceptable Prediction Zone Method. Journal of Food Protection, 70(2), pp.M129-M137.

Pakravan, M., Heuzey, M.-C. \& Ajji, A., 2011. A fundamental study of chitosan/PEO electrospinning. Polymer, 52(21), pp.4813-4824.

Pakravan, M., Heuzey, M.-C. \& Ajji, A., 2012a. Core - Shell Structured PEOChitosan Nanofibers by Coaxial Electrospinning. Biomacromolecules, 13, pp.412-421.

Pakravan, M., Heuzey, M.-C. \& Ajji, A., 2012b. Determination of Phase Behavior of Poly(ethylene oxide) and Chitosan Solution Blends Using Rheometry. Macromolecules, 45(18), pp.7621-7633. Available at: http://dx.doi.org/10.1021/ma301193h.

Paluszkiewicz, C. et al., 2011. FT-IR study of montmorillonite-chitosan nanocomposite materials. Spectrochimica Acta - Part A: Molecular and Biomolecular Spectroscopy, 79(4), pp.784-788.

Rieger, K.A., Birch, N.P. \& Schiffman, J.D., 2016. Electrospinning chitosan/poly(ethylene oxide) solutions with essential oils: Correlating solution 
rheology to nanofiber formation. Carbohydrate Polymers, 139, pp.131-138.

Available at: http://dx.doi.org/10.1016/j.carbpol.2015.11.073.

Rinaudo, M. et al., 1999. Influence of acetic acid concentration on the solubilization of chitosan. Polymer, 40, pp.7029-7032. Available at:

http://www.cermav.cnrs.fr/monos/publi-pdf/P99-52.pdf.

Sakellariou, P., Abraham, M.H. \& Whiting, G.S., 1994. Solubility characteristics of poly(ethylene oxide): Effect of molecular weight, end groups and temperature. Colloid \& Polymer Science, 272(7), pp.872-875.

Sankalia, M.G. et al., 2007. Reversed chitosan-alginate polyelectrolyte complex for stability improvement of alpha-amylase: Optimization and physicochemical characterization. European Journal of Pharmaceutics and Biopharmaceutics, 65(2), pp.215-232.

Schatz, C. et al., 2003. Typical physicochemical behaviours of chitosan in aqueous solution. Biomacromolecules, 4, pp.641-648.

Schiffman, J.D. \& Schauer, C.L., 2007. Cross-linking chitosan nanofibers. Biomacromolecules, 8(2), pp.594-601.

Schubert, M., Muffler, A. \& Mourad, S., 2012. The use of a radial basis neural network and genetic algorithm for improving the efficiency of laccase-mediated dye decolourization. Journal of Biotechnology, 161(4), pp.429-436. Available at: http://dx.doi.org/10.1016/j.jbiotec.2012.08.003.

Shenoy, S.L. et al., 2005. Role of chain entanglements on fiber formation during electrospinning of polymer solutions: Good solvent, non-specific polymerpolymer interaction limit. Polymer, 46(10), pp.3372-3384.

Silva, S.M.L. et al., 2012. Application of Infrared Spectroscopy to Analysis of Chitosan / Clay Nanocomposites. In Infrared spectroscopy-materials science, engineering and technology. pp. 43-62.

Spasova, M. et al., 2004. Preparation of chitosan-containing nanofibres by electrospinning of chitosan/poly(ethylene oxide) blend solutions. E-Polymers, (56), pp.1-12.

Tchemtchoua, V.T. et al., 2011. Development of a Chitosan Nanofibrillar Scaffold for Skin Repair and Regeneration. Biomacromolecules, 12(9), pp.3194-3204.

Tegl, G. et al., 2016. Antimicrobial Cellobiose Dehydrogenase-Chitosan Particles. ACS Applied Materials and Interfaces, 8(1), pp.967-973.

Thirugnanasambandham, K. \& Sivakumar, V., 2016. Preparation of Chitosan based Nanofibers: Optimization and Modeling. International Journal of Chemical Reactor Engineering, 14(1), pp.283-288. Available at: http://www.degruyter.com/view/j/ijcre.2016.14.issue-1/ijcre-2015-0029/ijcre2015-0029.xml.

Uyar, T. \& Besenbacher, F., 2008. Electrospinning of uniform polystyrene fibers: The effect of solvent conductivity. Polymer, 49(24), pp.5336-5343. Available at: http://dx.doi.org/10.1016/j.polymer.2008.09.025.

Wang, Q.Z. et al., 2006. Protonation constants of chitosan with different molecular weight and degree of deacetylation. Carbohydrate Polymers, 65(2), pp.194-201.

Wang, W. et al., 1991. Determination of the Mark-Houwink equation for chitosans with different degrees of deacetylation. International Journal of Biological Macromolecules, 13(5), pp.281-285.

Wang, X. et al., 2015. Controlled release of protein from core-shell nanofibers prepared by emulsion electrospinning based on green chemical. Journal of 
Applied Polymer Science, 132(16), pp.1-9.

Wei, M. et al., 2006. Core-sheath structure in electrospun nanofibers from polymer blends. Macromolecular Materials and Engineering, 291(11), pp.1307-1314.

Yang, Y.M. et al., 2007. The controlling biodegradation of chitosan fibers by Nacetylation in vitro and in vivo. Journal of Materials Science: Materials in Medicine, 18(11), pp.2117-2121.

Yazgan, G. et al., 2015. Tunable release of hydrophilic compounds from hydrophobic nanostructured fibers prepared by emulsion electrospinning. Polymer, 66, pp.268-276. Available at:

http://www.sciencedirect.com/science/article/pii/S0032386115003821 [Accessed July 25, 2016].

Yoshihara, T., Tadokoro, H. \& Murahashi, S., 1964. Normal Vibrations of the Polymer Molecules of Helical Conformation. IV. Polyethylene Oxide and Polyethylene-d4 Oxide. The Journal of Chemical Physics, 41(9), pp.2902-2911. Available at: http://link.aip.org/link/JCPSA6/v41/i9/p2902/s1\&Agg=doi\%5Cnhttp://scitation.aip. org/content/aip/journal/jcp/41/9/10.1063/1.1726373.

Zhang, J.F. et al., 2009. Electrospun core-shell structure nanofibers from homogeneous solution of poly(ethylene oxide)/chitosan. Macromolecules, 42(14), pp.5278-5284.

Zhang, Y.-S. et al., 2012. Memory effect on solution crystallization of high molecular weight poly(ethylene oxide). Polymer, 53(16), pp.3621-3628. Available at: http://linkinghub.elsevier.com/retrieve/pii/S0032386112005010.

Zhang, Y.Z. et al., 2008. Chitosan nanofibers from an easily electrospinnable UHMWPEO-doped chitosan solution system. Biomacromolecules, 9(1), pp.136141.

Zhou, X., Kong, M., Cheng, X.J., et al., 2014. In vitro and in vivo evaluation of chitosan microspheres with different deacetylation degree as potential embolic agent. Carbohydrate Polymers, 113, pp.304-313. Available at: http://dx.doi.org/10.1016/j.carbpol.2014.06.080.

Zhou, X., Kong, M., Cheng, X., et al., 2014. Investigation of acetylated chitosan microspheres as potential chemoembolic agents. Colloids and Surfaces B: Biointerfaces, 123, pp.387-394. Available at: http://dx.doi.org/10.1016/j.colsurfb.2014.07.044.

Zhou, X. et al., 2013. Optimization and characteristics of preparing chitosan microspheres using response surface methodology. Journal of Applied Polymer Science, 127(6), pp.4433-4439.

Zhu, A.P., Zhao, F. \& Fang, N., 2008. Regulation of vascular smooth muscle cells on poly(ethylene terephthalate) film by O-carboxymethylchitosan surface immobilization. Journal of Biomedical Materials Research - Part A, 86(2), pp.467-476. 
Table 1: Parameters and their coded values used in the experimental design.

\begin{tabular}{lcccc}
\hline & Coded values & -1 & $\mathbf{0}$ & $\mathbf{1}$ \\
Parameters & 75 & 85 & 95 \\
\hline Deacetylation degrees of chitosan (\%) & 20 & 50 & 100 \\
Viscosity of chitosan (mPa*s) & 200 & 600 & 1,000 \\
Molecular weight of PEO (kDa) & & & 90 \\
Acetic acid percentage (wt \%) & 70 & 80 & $5 / 95$ \\
\hline
\end{tabular}


Table 2: Coefficient analysis ( $\mathrm{N}$ atomic surface composition [\%]).

\begin{tabular}{|c|c|c|}
\hline & Coefficients & \\
\hline Response mean & $\beta 0$ & $3.5800^{* *}$ \\
\hline DD [\%] & $\beta 1$ & $0.0919^{\text {n.s. }}$ \\
\hline Mw Chi [mPa*s] & $\beta 2$ & $-0.5475^{* *}$ \\
\hline Mw PEO [kDa] & $\beta 3$ & $-0.0219^{\text {n.s. }}$ \\
\hline PEO/Chi [\%] & $\beta 4$ & $2.2450^{* *}$ \\
\hline $\mathrm{AcOH}[\%]$ & $\beta 5$ & $0.1300^{\text {n.s. }}$ \\
\hline $\mathrm{DD}[\%]^{2}$ & $\beta 6$ & $0.0052^{\text {n.s. }}$ \\
\hline Mw Chi $\left[\mathrm{mPa}^{*} \mathrm{~s}\right]^{2}$ & $\beta 7$ & $0.1410^{\text {n.s. }}$ \\
\hline Mw PEO $[k D a]^{2}$ & $\beta 8$ & $-0.8748^{* *}$ \\
\hline PEO/Chi [\%] ${ }^{2}$ & $\beta 9$ & $-0.2656^{*}$ \\
\hline $\mathrm{AcOH}[\%]^{2}$ & $\beta 10$ & $0.0460^{\text {n.s. }}$ \\
\hline DD [\%]* Mw Chi [mPa*s] & $\beta 11$ & $-0.0650^{\text {n.s. }}$ \\
\hline DD [\%] Mw PEO [kDa] & $\beta 12$ & $-0.2700^{\text {n.s. }}$ \\
\hline DD [\%] ${ }^{*}$ PEO/Chi [\%] & $\beta 13$ & $0.0100^{\text {n.s. }}$ \\
\hline $\mathrm{DD}[\%]^{*} \mathrm{AcOH}[\%]$ & $\beta 14$ & $0.0175^{\text {n.s. }}$ \\
\hline Mw Chi $\left[\mathrm{mPa}^{*}\right]^{*} \mathrm{Mw}$ PEO [kDa] & $\beta 15$ & $0.0050^{\text {n.s. }}$ \\
\hline Mw Chi [mPa*s] ${ }^{*} \mathrm{PEO} / \mathrm{Chi}[\%]$ & $\beta 16$ & $-0.4300^{*}$ \\
\hline Mw Chi $\left[\mathrm{mPa}^{*} \mathrm{~s}\right]^{*} \mathrm{AcOH}[\%]$ & $\beta 17$ & $0.2100^{\text {n.s. }}$ \\
\hline Mw PEO [kDa] ${ }^{*}$ PEO/Chi [\%] & $\beta 18$ & $0.6475^{* *}$ \\
\hline Mw PEO $[k D a]^{*} \mathrm{AcOH}[\%]$ & $\beta 19$ & $0.0300^{\text {n.s. }}$ \\
\hline $\mathrm{PEO} / \mathrm{Chi}[\%]^{\star} \mathrm{AcOH}[\%]$ & $\beta 20$ & $0.8475^{* *}$ \\
\hline
\end{tabular}

${ }^{*}=$ significant $(P<0.05) ;{ }^{* *}=$ highly significant $(P<0.001)$; n.s.=not significant $(P \geq 0.05)$ 
Table 3: Atomic composition of the three selected samples as determined by XPS, elemental analysis and theoretical calculation.

\begin{tabular}{c|cccc|l}
\hline $\begin{array}{c}\text { Chi/PEO } \\
\text { [wt/wt \%] }\end{array}$ & $\mathbf{5}$ & $\mathbf{C}$ & $\mathbf{N}$ & $\mathbf{H}$ & \\
\hline \multirow{4}{*}{$70 / 30$} & 46.1 & 39.3 & 7.5 & 7.0 & Theoretical \\
& 44.7 & 42.2 & 5.1 & 7.9 & Elemental analysis \\
& 66.7 & 32.8 & 0.5 & - & XPS \\
\hline \multirow{4}{*}{$82.5 / 17.5$} & 45.4 & 39.5 & 8.1 & 7.0 & Theoretical \\
& 42.5 & 43.3 & 6.3 & 7.6 & Elemental analysis \\
& 67.4 & 28.8 & 3.8 & - & XPS \\
\hline \multirow{3}{*}{$95 / 5$} & 45.3 & 39.6 & 8.3 & 6.8 & Theoretical \\
& 40.9 & 44.9 & 6.7 & 7.4 & Elemental analysis \\
& 65.0 & 29.1 & 6.0 & - & XPS \\
\hline
\end{tabular}

